\title{
Plasma-lithium interaction in the CDX-U spherical torus
}

\author{
G.Y. Antar a,*, R.P. Doerner ${ }^{a}$, R. Kaita ${ }^{b}$, R. Majeski ${ }^{\text {b }}$, J. Spaleta ${ }^{\text {b }}$, \\ T. Munsat ${ }^{\mathrm{b}}$, B. Jones ${ }^{\mathrm{b}}$, R. Maingi ${ }^{\mathrm{c}}, \mathrm{V}$. Soukhanovskii ${ }^{\mathrm{b}}, \mathrm{H}$. Kugel ${ }^{\mathrm{b}}$, \\ J. Timberlake ${ }^{\text {b }, ~ S . I . ~ K r a s h e n i n n i k o v ~}{ }^{\text {a }}$, S.C. Luckhardt ${ }^{\text {a }}$, R.W. Conn ${ }^{\text {a }}$

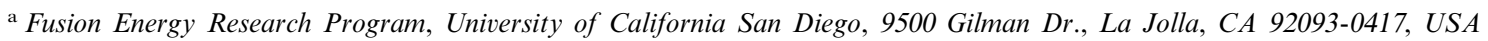 \\ b Princeton Plasma Physics Laboratory, Princeton, NJ, USA \\ c Oak Ridge National Laboratory, Oak Ridge, TN, USA
}

Accepted 15 February 2002

\begin{abstract}
Results on the interaction between plasma in the current drive experiment-upgrade (CDX-U) spherical torus and a liquid lithium limiter are reported. It is observed that macroscopic lithium droplets detach from the limiter head and fall towards the plasma core. However, no disruptions occurred during these discharges despite the fact that relatively large-scale blobs are observed entering the confined plasma. A multi-tip Langmuir probe measures the edge plasma properties. It is found that the average density and temperature and their fluctuations are unaffected by the presence of lithium within experimental error. (C) 2002 Elsevier Science B.V. All rights reserved.
\end{abstract}

Keywords: Plasma-material interaction; Lithium walls; MHD effects; CDX-U

\section{Introduction}

Putting liquid lithium in contact with the plasma is one direction being explored in the development of new concepts for limiters and divertor target plates in magnetic confinement devices. The motivation is to avoid some wellknown problems of plasma-facing components such as surface erosion and blistering. In addition, a flowing liquid should allow efficient heat removal [1] and may permit density control and power extraction. [2,3] In addition, liquid lithium

\footnotetext{
* Corresponding author. Tel.: + 1-858-822-4116; fax: + 1858-534-7716.

E-mail address: gantar@ferp.ucsd.edu (G.Y. Antar).
}

can provide tritium accumulation control [4].

Wall conditioning by lithium evaporation into the tokamak vessel was first performed with good results in TFTR [5]. Using lithium pellet in combination with high power neutral beam injection enabled the TFTR tokamak to achieve high plasma performance [6]. Evaporating lithium to getter the inside walls of a tokamak was also done in the TdeV [7] and JIPP T-IIU tokamaks [8]. However, unlike in TFTR, no significant confinement enhancement was reported in these devices, although the role of lithium to reduce impurities in the plasma was confirmed. In particular, the JIPP T-IIU experiments confirmed the ability of lithium to getter different types of impurities such as $\mathrm{CO}$ and $\mathrm{CH}_{4}[8,9]$. 
Recently, in a series of experiments in the PISCES edge plasma simulation device, liquid lithium samples were exposed to plasma with different fluences. It was found, that liquid lithium has an erosion rate higher than what is expected from standard sputtering, such as predicted by the results of Laszlo and Eckstein [10].

In the present paper, we report on liquid lithium interactions with confined plasmas. A limiter head, wetted with liquid lithium, is inserted into the plasma of the current drive experimentupgrade (CDX-U) spherical torus [11]. As described in Section 2, where the experimental setup is presented, the quantity of lithium in contact with the plasma may be of the order of a few $\mathrm{cm}^{3}$. The lithium behavior is analyzed by images using a fast camera [12]. During the plasma interaction with the limiter, the plasma edge is characterized by a Langmuir probe. While no dramatic effects on edge density and temperature were recorded, the lithium is observed to form droplets that detach from the limiter, mainly due to electromagnetic forces. No plasma disruptions were linked to the presence of lithium droplets in the confined plasma, even though the droplets are macroscopic in dimension.

\section{Experimental setup}

A lithium limiter is installed on the CDX-U spherical torus. The plasma has the following parameters: toroidal magnetic field $B=2.3 \mathrm{kG}$; plasma current $I_{\mathrm{p}}$ up to $60 \mathrm{kA}$; major and minor radii, respectively equal to 34 and $22 \mathrm{~cm}$. The plasma is elongated, with a height between 35 and $38 \mathrm{~cm}$. A typical plasma discharge is described in Fig. 1, where the evolution of the line-averaged density as well as edge temperature and density is shown; the plasma current and the currents induced to flow in the limiter are also plotted.

The limiter is a heated stainless-steel cylinder covered with a fine stainless-steel mesh $(20 \times 20$ $\mathrm{cm}^{-2}$ ) to increase the liquid adhesion onto the metal. Lithium on the limiter is liquefied using heating elements inside the cylinder. Wetting is achieved for the lithium covering the whole surface of the limiter, that is, approximately $300 \mathrm{~cm}^{2}$.
The limiter head is linked to a lithium reservoir by a tube that passes through the cylinder center. The reservoir is located within the limiter support shaft. Its temperature is more or less independent of the limiter temperature as the head is not in close thermal contact and is heated separately. The entire setup can be retracted to the outside of the confinement vessel and separated from the plasma by the means of two gate valves. Fig. 2 illustrates the setup. The limiter head is positioned at $16.8 \mathrm{~cm}$ above the mid-plane, and has a radius of $2.5 \mathrm{~cm}$ and a length of $19.6 \mathrm{~cm}$. Since the average lithium thickness is $0.5 \mathrm{~mm}$, the quantity of lithium introduced into the vacuum chamber is of the order of $15 \mathrm{~cm}^{3}$. However, only a part of the lithium gets in contact with the plasma edge. This leads to an effective volume of $2 \mathrm{~cm}^{3}$, with a surface of the order of $20 \mathrm{~cm}^{2}$ and an average thickness of $1 \mathrm{~mm}$.

In order to make a plasma with $\sim 10 \mathrm{~ms}$ pulse duration and relatively high current $\left(I_{\mathrm{p}} \sim 50 \mathrm{kA}\right)$, surface wall and lithium conditioning at the beginning of the day was found to be necessary [12]. An argon glow discharge, using the limiter head
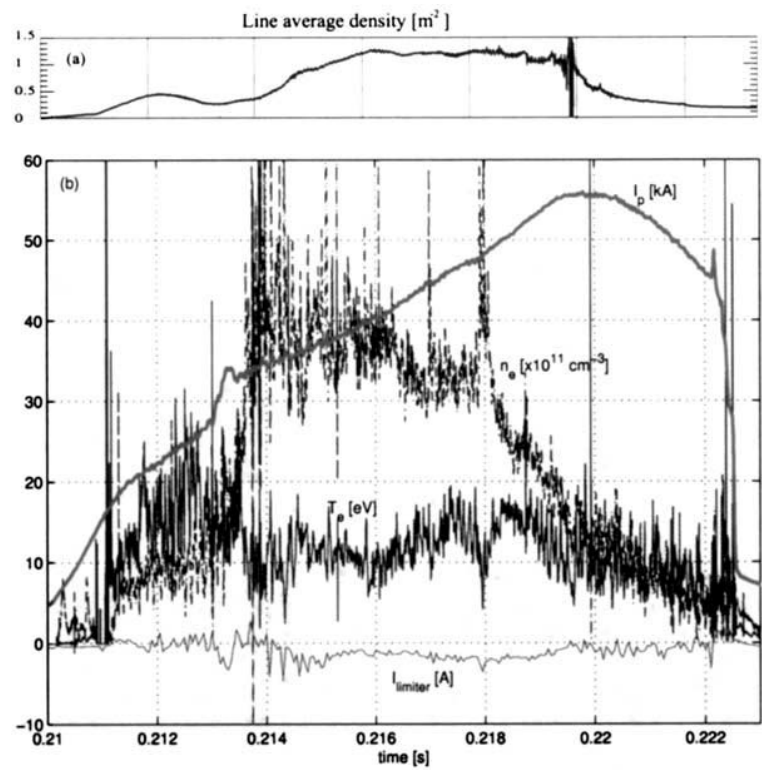

Fig. 1. Typical plasma parameters in the CDX-U spherical torus showing the line averaged density $\left(\times 10^{19} \mathrm{~m}^{-2}\right)$ in (a) as well as edge density and temperature in (b) where plasma $\left(I_{\mathrm{p}}\right)$ and lithium limiter current $\left(I_{\text {limiter }}\right)$ are also plotted. 


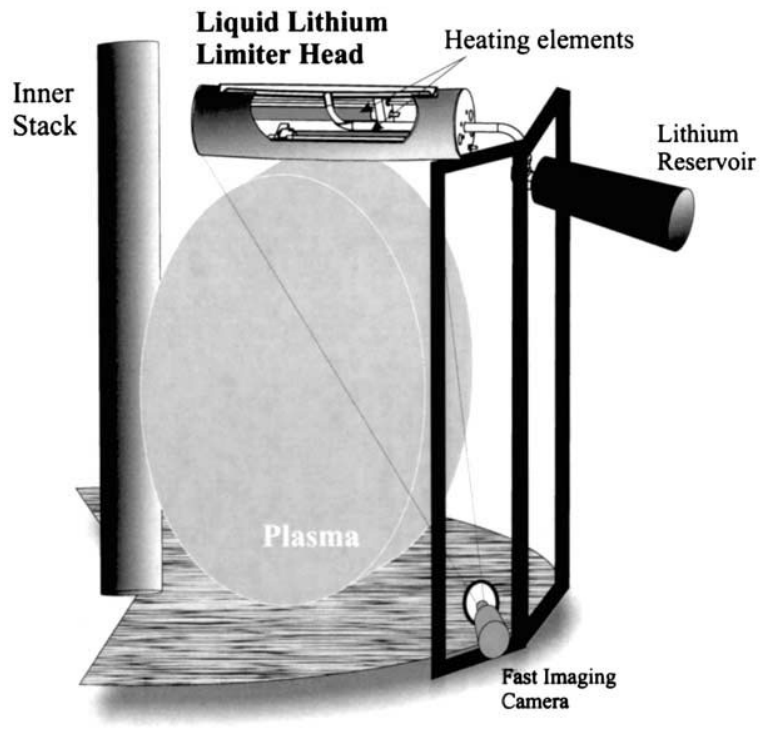

Fig. 2. Illustration of the limiter head in the CDX-U confinement chamber. Note the tube which links the limiter head to the reservoir inside the cylinder. The diagnostic camera is also illustrated looking from below onto the head.

as the cathode, for about $1 \mathrm{~h}$ was verified to be sufficient to remove impurities out of the lithium in the liquid state. Visually, the glow discharge transforms the color of the lithium on the limiter head to shiny metallic from matte gray with dark patches. Then, conditioning the walls is also necessary and this is achieved by standard titanium gettering. The lithium limiter is withdrawn from the vessel during this gettering procedure. Following these conditionings, it was possible to maintain the same plasma scenario throughout each run day, consisting of approximately 40 shots.

\section{Lithium-plasma interaction}

In this section, we describe the interaction between the lithium limiter and the plasma. A Kodak SR Ultra Motion Corder Analyzer is installed behind a view port and focused on the limiter from below (see illustration in Fig. 2). It has a scanning frequency of $1 \mathrm{kHz}$, a shutter speed of $50 \mu \mathrm{s}$ and a resolution of $256 \times 240$ pixels. A lithium-I filter centered at $670.8 \mathrm{~nm}$ is installed to detect the line radiation from excited neutral lithium atoms. Fig. 3 shows the time evolution of the plasma current and snapshots of the limiter while the lithium is in the liquid state. We note that the plasma can have a 'hard' interaction (sub-Figs. 3 and 4) where macro-scale lithium droplets occur or 'soft' interaction (sub-Figs. 1, 5 and 6) where droplets are not observed. The lack of feedback on the plasma vertical position may be the cause of this non-stationary behavior.

\subsection{Causes for liquid lithium detachment from the limiter}

In order to assess the cause of the lithium droplet release from the limiter towards the plasma, it is necessary to estimate the different forces acting on the lithium. A rather comprehensive discussion of the particle and heat fluxes effects on limiters can be found in Ref. [13]. In what follows, we show only those pressure terms that are relevant to our experiment, which are as follows:

- Gravitational pressure, $P_{g}=p g h$, is about 50 dyne $\mathrm{cm}^{-2}$ for a density $\rho=0.53 \mathrm{~g} \mathrm{~cm}^{-3}$, a gravitation acceleration $g=10^{3} \mathrm{~cm} \mathrm{~s}^{-2}$ and a height $h$ of $1 \mathrm{~mm}$. This pressure destabilizes the liquid and tends to make it fall.

- The plasma pressure, $P_{p}=2 n T$, is found to be between 30 and 300 dyne $\mathrm{cm}^{-2}$ for an edge plasma density $n \cong 10^{12}-10^{13} \mathrm{~cm}^{-3}$ and a temperature $T=10 \mathrm{eV}$. This term is stabilizing, as it pushes the lithium on the mesh.

- The parallel momentum pressure, $P_{\mathrm{M}}=2 \Phi / v_{\|}$, known also as the plasma wind [13,14], caused by ions collision onto the lithium surface; $\Phi=$ $\gamma n c_{\mathrm{s}} T$ is the heat flux and $v_{\|}$is the parallel velocity. The plasma density and temperature are $n$ and $T, c_{\mathrm{s}}$ is the sound speed and $\gamma$ is the sheath transmission factor taken equal to 7 . This is a destabilizing force as it tends to make the lithium detach from the limiter and move in the toroidal direction. Given the density and temperature in the edge of CDX-U, this term is about 70 dyne $\mathrm{cm}^{-2}$.

- The surface pressure, $P_{\mathrm{s}}=\sigma / R$, acts against any surface deformation and is thus stabilizing. The surface tension coefficient, $\sigma$, is equal to 
about 398 dyne $\mathrm{cm}^{-1}$. For a curvature, $R$, between 2 and $4 \mathrm{~cm}, P_{\mathrm{s}}$ is between 100 and 200 dyne $\mathrm{cm}^{-2}$.

- Because the limiter is grounded, an electromagnetic $\vec{j} \times \vec{B}$ force acts on the lithium creating a pressure $P_{j}=j B / A$. The current density, $j$, is estimated from the current drawn by the limiter, while $B$ is the toroidal magnetic field and $A$ is the surface area perpendicular to $\vec{B}$ on which the electromagnetic pressure is exerted. The liquid lithium sticks to the limiter fine mesh, giving rise to micron scales everywhere except at the central zone. The lithium height at the center can be as large as $1 \mathrm{~mm}$, and is caused by the lithium weight as it comes in equilibrium with the lithium surface tension. In this region, assuming the current is perpendicular to the lithium surface, one finds $P_{\mathrm{j}}$ to be $(2.5-5) \times 10^{3}$ dyne $\mathrm{cm}^{-2}$. This pressure is of course destabilizing, as it tends to force the liquid in the poloidal direction, assuming that the current in the liquid lithium flows in the radial direction.

Accordingly, we find that the $\vec{j} \times \vec{B}$ force dominates the dynamics of the lithium and can cause large amounts of liquid (in the form of droplets) to detach from the limiter, as observed in these experiments.

Another interesting aspect associated with Fig. 3 is the nature of the lithium droplets reaching the

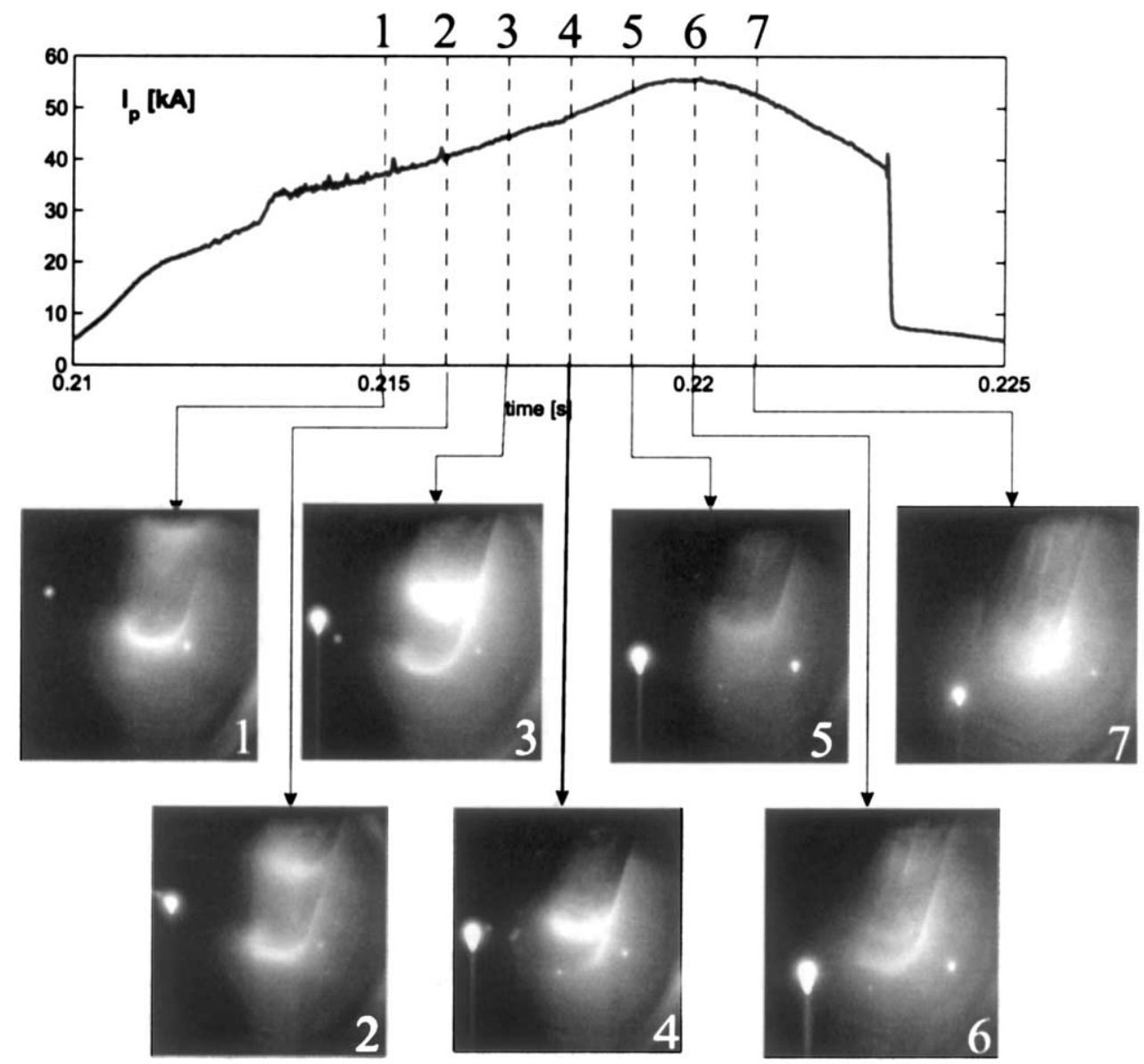

Fig. 3. Seven consecutive camera images labeled from 1 to 7 of LiI light coming from the limiter head and surroundings. The plasma current is also shown to indicate when in the discharge the images are taken. One can notice two macroscopic droplets of lithium on either side of the limiter. 


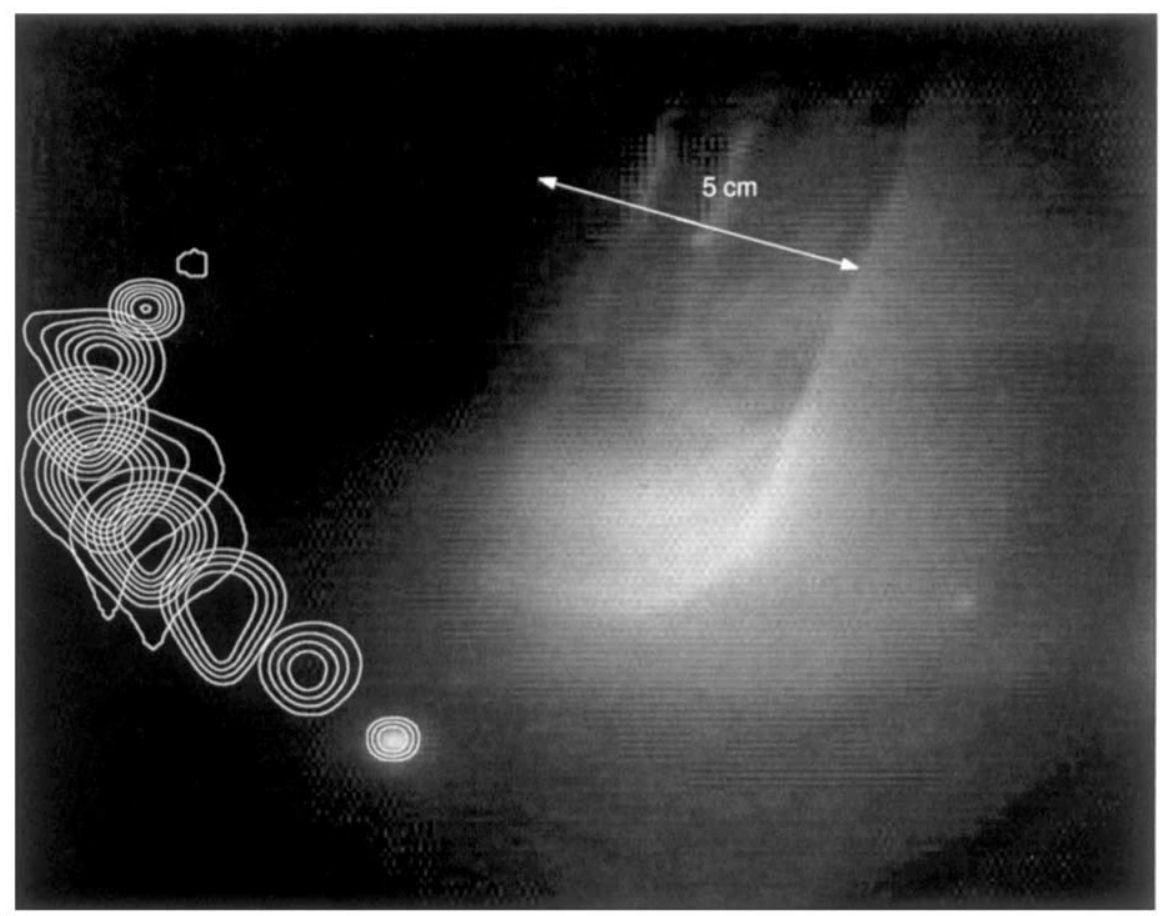

Fig. 4. The large droplet contour plot on the left side of the limiter inside the vessel is superimposed on one image so one can see the motion. The arrow indicates the width of the limiter head.

plasma. Detached from the limiter, the droplet diameter is estimated about $0.5 \mathrm{~cm}$, and its sharp edges indicate that the camera is focused on it. It is thus located at approximately the same distance from the camera as the limiter. In Fig. 4, the motion of the droplet is tracked and plotted in the same figure. The droplet path describes an arc, strongly suggesting that it is following a magnetic field line. From the first image to the last, the droplet has moved about $10 \mathrm{~cm}$, leading to an estimated toroidal velocity about $1000 \mathrm{~cm} \mathrm{~s}^{-1}$.

When the droplet detaches from the limiter, it is submitted mainly to gravity and plasma wind. The force of gravity is $F_{g}=M_{\mathrm{D}} g$, where $M_{\mathrm{D}}$ is the droplet mass and $g$ is the gravity acceleration. This force is of the order of 15 dyne. The force of the plasma wind is $F_{\mathrm{M}}=P_{\mathrm{M}} A$. It is estimated for a density about $10^{13} \mathrm{~cm}^{-3}$, an electron temperature of $100 \mathrm{eV}$ and $A \cong 0.12$ $\mathrm{cm}^{2}$ leading to an order of magnitude about 7000 dyne. It is much greater than the gravity force causing the droplet to move along the field lines as it is observed in Fig. 4. The velocity that can be achieved subject to this parallel momentum force can be estimated by assuming that the acceleration is constant during the droplet travel in the plasma and the initial velocity is null. This leads to a droplet velocity about $400 \mathrm{~cm} \mathrm{~s}^{-1}$ after staying $7 \mathrm{~ms}$ in the plasma. This number is of the order of magnitude of the droplet velocity detected by the several snapshots of the camera.

\subsection{Survival of large lithium droplets in the plasma}

One might be surprised to see that such a macroscopic droplet with radius about $0.2 \mathrm{~cm}$ can survive, as it is subject to plasma heat and particle fluxes. The heat flux per unit area on the droplet surface can be divided into two contributions, one caused by ions $(\Phi)$ and the other by electrons (ohmic heating). They lead to an 
increase of the droplet temperature according to $\rho c_{\mathrm{p}} V \partial T / \partial t$, where the surface cooling due to evaporation is neglected. Here $c_{\mathrm{p}}$ is the specific heat at constant pressure, and $V$ and $\rho$ are respectively the volume and density of the lithium droplet. Taking into account that for lithium, $\rho=0.53$ [g $\left.\mathrm{cm}^{-3}\right]$ and $c_{\mathrm{p}}=3.6\left[\mathrm{~J} \mathrm{~g}^{-1} \mathrm{~K}^{-1}\right]$, and assuming $T \sim 100 \mathrm{eV}, n \sim 10^{12} \mathrm{~cm}^{-3}$, and the sheath transmission factor $\gamma=7$, one finds that the ion flux leads to a temperature increase of $\partial_{t} T \sim 10^{4} \mathrm{~K} \mathrm{~s}^{-1}$ for a droplet with a radius of $0.2 \mathrm{~cm}$. On the other hand, the ohmic power of the plasma is about 100 $\mathrm{kW}$ since $I_{\mathrm{p}}=50 \mathrm{kA}$ and the loop voltage is about $2 \mathrm{~V}$. A droplet with $0.2 \mathrm{~cm}$ of radius intercepts about $1 / 100$ of the total ohmic power, that is, on the order of $1 \mathrm{~kW}$. This is slightly greater than the heating caused by the ions which is about $800 \mathrm{~W}$.

As such, the total heating power to the droplet is about $2 \mathrm{~kW}$ and this leads to a temperature increase of about $200 \mathrm{~K}$ in $10 \mathrm{~ms}$. At the vessel pressure about $10^{-4} \mathrm{~Pa}$, the droplet temperature would be about the boiling point which leads to its evaporation. The evaporation rate has the following expression

$Q_{\text {evap }}=4.37 \times 10^{-3} P_{\text {sat }}(M / T)^{1 / 2}\left(\mathrm{~kg} \mathrm{~m}^{-2} \mathrm{~s}^{-1}\right)$,

where $M$ is the atomic weight and $P_{\text {sat }}$ is the saturated vapor pressure deduced from the expression [9]

$\log _{10} P_{\text {sat }}=9.7641-8502.7 / T-1.174 \log _{10} T$.

Putting the numbers in the above expressions, we find that in $10 \mathrm{~ms}$ a negligible amount of the droplet $\left(10^{-30} \%\right)$ is expected to evaporate. This is consistent with our observation that the macroscopic droplet survives.

We have also assessed the droplet erosion rate due to sputtering by the incident ion flux. The yield per incident ion, estimated by Doerner et al., is about $10 \%$ [10]. The number of lithium atoms leaving the droplet due to erosion in $10 \mathrm{~ms}$ is thus about $10^{16}$ atoms. This amount is negligible compared to the total number of atoms in a lithium droplet with a $2 \mathrm{~mm}$ radius, namely $10^{21}$. So again we conclude that millimeter size droplets are expected to survive throughout the discharge duration in CDX-U, as they are observed to do.

\subsection{Evidence of a large number of small lithium droplets}

Above, we discussed the dynamics of a macroscopic lithium droplet. However, as one might have suspected from Fig. 3, the light intensity coming from the limiter might as well be caused by small-scale droplets. Fig. 5, taken with the camera focused on the plasma just below the limiter, indicates the existence of such a large number of small-scale lithium droplets with submillimetric dimension. They are ionized very close to the plasma edge and most probably evaporate rapidly because of their small size. In the first four images, the droplets have rather circular shape indicating that their velocity is small and thus their motion is well resolved. In the last two images, one notices stream-like structures reflecting the motion of the lithium in the $50 \mu$ s exposure time. An estimated velocity of $2 \times 10^{4} \mathrm{~cm}$ $\mathrm{s}^{-1}$ can be deduced from the images in the figure.

Droplets being released from the limiter were often observed. And yet, at the end of the day, or after about 40 shots, no significant decrease of the amount of lithium on the limiter head was recorded. The reason lies in the fact that the tube inside the limiter (which links the head to the reservoir) is heated to about $300{ }^{\circ} \mathrm{C}$. A significant amount of lithium in the tube and at the upper end of the reservoir are thus in a liquid state. We believe that as droplets are ejected from the limiter, the capillary force allows fresh liquid lithium from the tube onto the reservoir and supplies the limiter head in a self-regulated way [15].

Although a large number of macroscopic droplets of lithium are observed to leave the limiter head, no effect on plasma stability is observed. In particular, no plasma disruptions were recorded. This result is in agreement with that from the TdeV tokamak experiment where a rather small amount of lithium was used [7]. Moreover, it is worth mentioning that the physics behind the droplets formation is not yet understood. It could be hydrodynamic instability caused by the $\vec{j} \times \vec{B}$ force or the plasma wind or a coupling between these two effects. Unless the formation process is well understood we do not a have a way to control their frequency or sizes. 


\section{Edge properties as function of the distance to the last closed flux surface}

The effect of a liquid lithium limiter on plasma performance is assessed using a three-tip Langmuir probe to measure edge ion saturation current, the floating potential and the electron temperature. The probe is located at the midplane at a toroidal angle $4 \pi / 3$ downstream from the limiter [12]. The frequency of the data acquisition is $1 \mathrm{MHz}$. All the shots were performed under identical discharge conditions. Edge properties are recorded first without the limiter inside the vessel. Later, the limiter was inserted and heated to $250{ }^{\circ} \mathrm{C}$. The current drawn to the limiter is the same for all discharges, indicating an overall consistent plasma interaction with the limiter.

It is found that edge plasma density and temperatures are not significantly affected by the presence of the liquid lithium limiter. The data in Fig. 6(a) and (b) illustrates this result. The plasma density and temperature increase as we probe closer to the core plasma; but the same behavior is recorded with or without the lithium limiter. The variation in the turbulence level with respect to the major radius $R$ seems also to be nearly unaffected, as shown in Fig. 6(c) and (d). The same intensity dependence on the distance to the last closed flux surface is detected with or without the limiter.

The fact that no changes were recorded with or without the lithium limiter, and whether the lithium is in the liquid or solid state, may be for two reasons:

1. In addition to the lithium, plasma is also in contact with other limiters and most importantly the inner central CDX-U stack. This adds sources and sinks to the recycling budget at the edge and thus could decrease the effects of the lithium head. We should mention that no significant amount was present on the inner walls mainly because the lithium temperature on the limiter remained below evaporation.

2. Even in the case where the role of other recycling areas then the lithium limiter were neglected, the limiter head would intersect about $5 \%$ of the edge plasma. If one assumes that
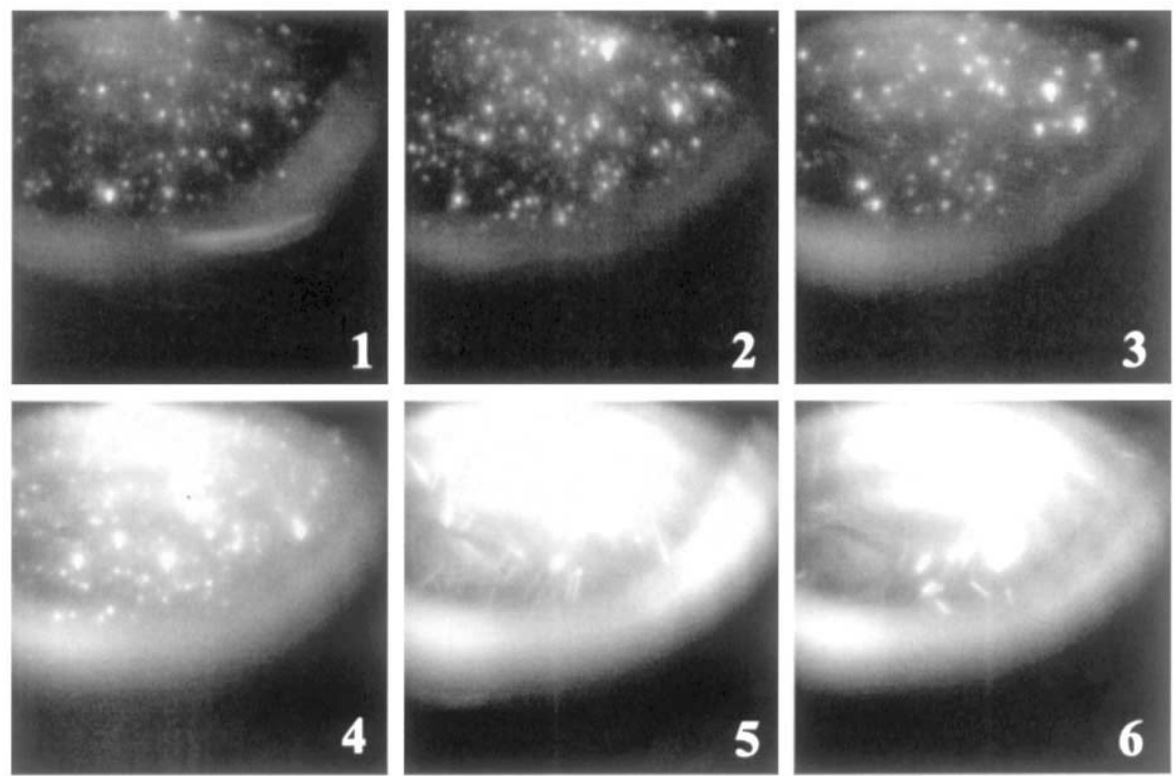

Fig. 5. Six consecutive LiI camera images of the plasma just below the limiter head. Images 1-4 show little motion of the small-scale droplets ejected from the limiter. During images 5 and 6 , the streaking nature of the droplets indicates ejection from the limiter with a much larger velocity. 

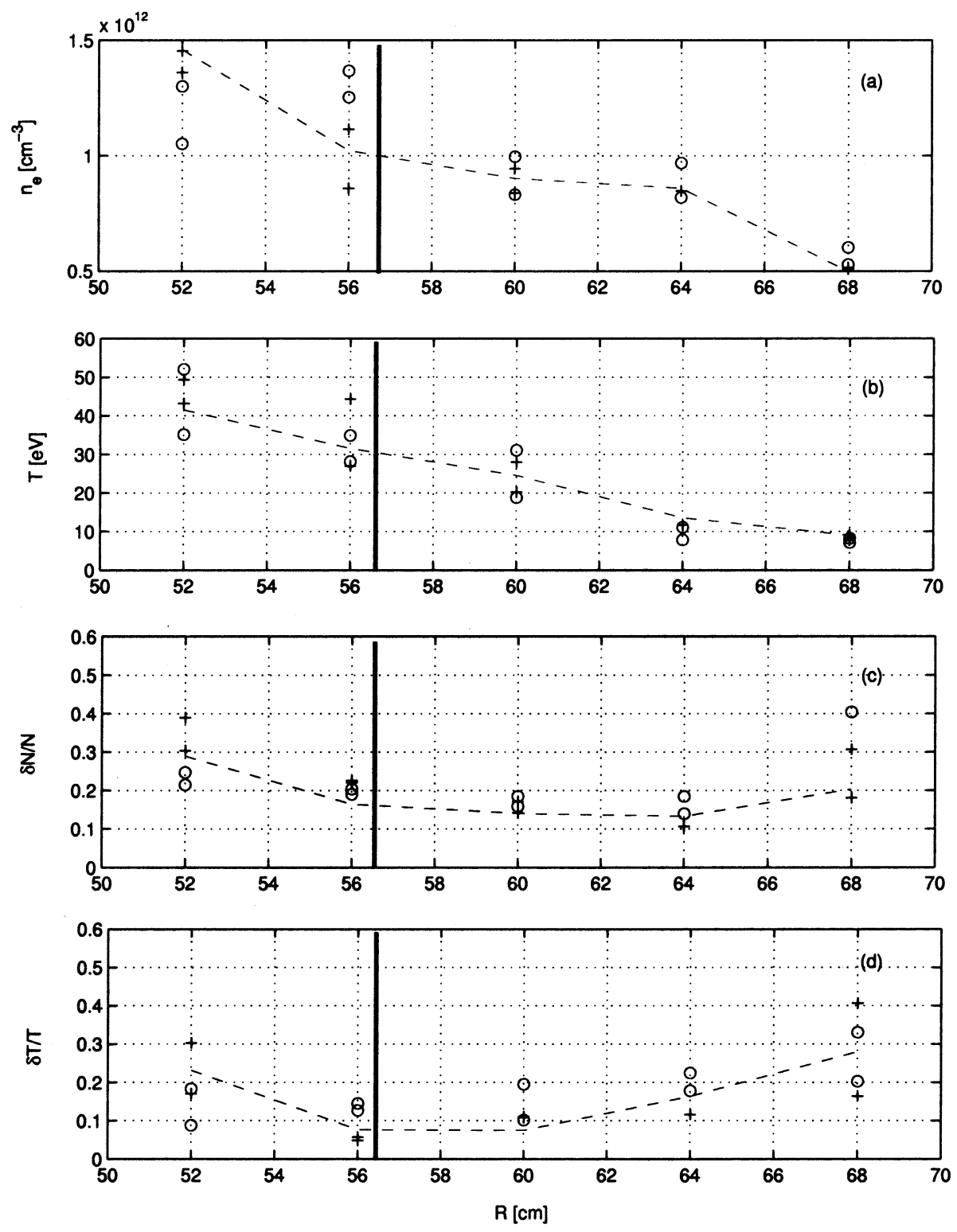

Fig. 6. In (a) and (b), the average values of the edge density and temperature are plotted. In (c) and (d), the relative turbulence intensity of edge density $(\delta n / n)$ and temperature $(\delta T / T)$ as function of $R$; ' + ' and 'o' denote respectively values taken when the lithium limiter is outside and inside the vessel. The vertical thick solid line denotes the last closed flux surface position.

$90 \%$ of the hydrogen intersecting the limiter is absorbed, this would lead to a change of about $4 \%$ in the edge density. This is much smaller than either the reproducibility of the discharges, estimated at about $10 \%$, or in the density fluctuations level, estimated to be about
$20 \%$. Future experiments will increase the contact area of lithium with the edge plasma significantly by installing a toroidal tray [11].

Other than characterizing the edge radial profiles, dedicated shots were performed as the lithium 
is heated to temperatures above the melting point. Here also, no significant changes were recorded for the plasma edge density, temperature and turbulence levels. These, each, remained nearly unchanged, within the $20 \%$ limit set by the fluctuation level and discharge reproducibility.

\section{Conclusion}

In this article, we addressed the problem of magnetohydrodynamic stability of liquid lithium surfaces as it comes in contact with the plasma. This issue is essential to allow flowing liquid lithium for divertor target to be used as a plasmafacing component in order to control, among other things, plasma density and power extraction. In the CDX-U spherical torus, we first established good plasma discharges with the lithium head inserted in the vessel identical to discharges without the lithium limiter. Then, the behavior of the plasma as it comes in contact with a liquid lithium limiter was studied.

Imaging the limiter allowed us to reveal the existence of lithium droplets detaching from the limiter and being of various sizes. These macroscopic droplets are ejected into the plasma with velocities ranging from few 10 s to $1000 \mathrm{~cm} \mathrm{~s}^{-1}$. In these experiments, the electromagnetic force $\vec{j} \times \vec{B}$ is sufficient to cause such behavior. Meanwhile, no disruptions took place because of the droplets being ejected and sent into the plasma. According to the results presented in this article, further dedicated experiments should be made to control the liquid metals in contact with the plasma especially as they are subject to electromagnetic forces as well as plasma wind.

For larger tokamaks where the magnetic field is about 10 Tesla, that is 50 times greater than in CDX-U, the results of this article show that the control of the electromagnetic forces is a crucial issue. One has to choose the right direction for the currents flowing in the liquid metal, by floating or grounding it in some particular way, so as the resulting force is stabilizing; one can also bias the liquid to produce a stabilizing force.

Experiments to characterize the plasma edge using a Langmuir probe showed that no change occurred in either the average or the fluctuations levels of density and temperature above experimental error even though droplets were entering the plasma. This is consistent with the estimated degree of interaction between the plasma and the limiter head. Hence, further experiments should address the density control problem by increasing the lithium-plasma interaction surface.

\section{Acknowledgements}

We are thankful to fruitful discussion with $\mathrm{N}$. Morley. This work was supported by the Department of Energy under the grants DE-FG0395ER-54301, DE-AC02-76CH-03073 and DE-AC05-00OR-22725.

\section{References}

[1] I.R. Kirillov, I.V. Danilov, S.I. Sidorenkov, Yu.S. Strebkov, R.F. Mattas, Y. Gohar, T.Q. Hua, D.L. Smith, Fus. Eng. Des. 39-40 (1998) 669.

[2] M.A. Abdou, T.A. Team, A. Ying, N. Morley, K. Gulec, S. Smolentsev, M. Kotschenreuther, S. Malang, S. Zinkle, T. Rognlien, P. Fogarty, B. Nelson, R. Nygren, K. McCarthy, M.Z. Youssef, N. Ghoniem, D. Sze, C. Wong, M. Sawan, H. Khater, R. Woolley, R. Mattas, R. Moir, S. Sharafat, J. Brooks, A. Hassanein, D. Petti, M. Tillack, M. Ulrickson, T. Uchimoto, Fus. Eng. Des. 54 (2001) 181 .

[3] B. van der Schaaf, K. Ehrlich, P. Fenici, A.A. Tavassoli, M. Victoria, Fus. Eng. Des. 48 (2000) 499.

[4] T. Takeda, A. Ying, M.A. Abdou, Fus. Eng. Des. 28 (1995) 278.

[5] the TFTR Group, J.A. Snipes, E.S. Marmar, J.L. Terry, M.G. Bell, R.V. Budny, K.W. Hill, D.L. Jassby, D.K. Mansfield, D.M. Meade, H.K. Park, A.T. Ramsey, J.D. Strachan, B.C. Staton, E.J. Synakowski, D.N. Ruzic, M. Shaheen, J. Nucl. Mater. 196-198 (1992) 686.

[6] D.K. Mansfield, K.W. Hill, J.D. Strachan, M.G. Bell, S.D. Scott, R. Budny, E.S. Marmar, J.A. Snipes, J.L. Terry, S. Batha, R.E. Bell, M. Bitter, C.E. Bush, Z. Chang, D.S. Darrow, D. Ernst, E. Fredrickson, B. Grek, H.W. Herrmann, A. Janos, D.L. Jassby, F.C. Jobes, D.W. Johnson, L.C. Johnson, F.M. Levinton, D.R. Mikkelsen, D. Mueller, D.K. Owens, H. Park, A.T. Ramsey, A.L. Roquemore, C.H. Skinner, T. Stevenson, B.C. Stratton, E. Synakowski, G. Taylor, A. von Halle, S. von Goeler, K.L. Wong, S.J.Z.T. Group, Phys. Plasmas 3 (1996) 1892. 
[7] B. Terreault, H.Y. Guo, R.W. Paynter, W.W. Zuzak, G. Abel, M. Ennaceur, J. -L. Gauvreau, E. Haddad, L. Leblanc, G.G. Ross, H.H. Mai, N. Richard, B.L. Stansfield, D. Kéroack, M. Caorlin, D.K. Owens, D. Mueller, P.H. La Marche, S. Pitcher, J. Nucl. Mater. 220-222 (1995) 1130.

[8] H. Sugai, H. Toyoda, K. Nakamura, K. Furuta, M. Ohori, K. Toi, S. Hirokura, K. Sato, J. Nucl. Mater. 220-222 (1995) 254.

[9] H. Sugai, M. Ohori, H. Toyoda, Vacuum 47 (1996) 981.

[10] R.P. Doerner, M.J. Baldwin, R.W. Conn, A.A. Grossman, S.C. Luckhardt, R. Seraydarian, G.R. Tynan, D.G. Whyte, J. Nucl. Mater. 290-29 (2001) 166.

[11] R. Kaita and R. Majeski and R. Doerner and G. Antar and J. Timberlake and J. Spaleta and D. Hoffman and B. Jones and T. Munsat and H. Kugel and G. Taylor and D.
Stutman and V. Sukhanovskii and R. Maingi and S. Molesa and P. Efthimion and J. Menard and M. Finkinthal and S. Luckhardt. Princeton Plasma Physics Laboratory Report PPPL-3551 (2001).

[12] R. Kaita, P. Efthimion, D. Hoffman, B. Jones, H. Kugel, R. Majeski, T. Munsat, S. Fartopoulos, G. Taylor, J. Timberlake, V. Soukhanovskii, D. Stutman, M. Iovea, M. Finkinthal, R. Doerner, S. Luckhardt, R. Maingi, R. Causey, Rev. Sci. Instrum. 72 (2001) 915.

[13] S.A. Cohen, R. Budny, G.M. McCracken, M. Ulrickson, Nucl. Fus. 21 (1981) 233.

[14] N.B. Morley, A.A. Gaizer, M.A. Abdou, Fus. Eng. Des. 28 (1995) 176.

[15] V.A. Evtikhin, I.E. Lyublinski, A.V. Vertkov, V.G. Belan, I.K. Konkashbaev, L.B. Nikandrov, J. Nucl. Mater. 271\&272 (1999) 396. 